\title{
Language and aging research: new insights and perspectives
}

https://doi.org/10.1515/lingvan-2019-0025

Received April 8, 2019; accepted April 8, 2019

\begin{abstract}
Our introduction to the special collection gives an overview of the research projects which were originally presented at the third CLARe network conference. We group the research under four cross-sectional topics that unite the different contributions: the data used in the research, the theoretical frameworks, the languages and varieties which are represented and the situational contexts which are examined. These projects represent the current state of research in this field and allows the reader to orient themselves within this diverse field but also leaves many questions open and provides impetus for future lines of research. The interaction and collaboration between diverse disciplines is the central aspect which unites all contributions to the special collection.
\end{abstract}

Keywords: language and aging; lifespan; health communication; language change; interactional linguistics; conversation analysis; corpus linguistics; psycholinguistics; sociolinguistics; computational linguistics.

"Encounters in Language and Aging Research" was the title of the third conference organized by and for the network Corpora for Language and Aging Research (CLARe3). The special collection gathers some of the papers presented at the network's third and fourth conferences (March 2017, Freie Universität Berlin and February 2019, University of Helsinki), along with invited contributions on related topics. Both conferences provided the opportunity to present challenges faced and recent progress in the field, and to take advantage of the variety of linguistic sub-disciplines in which topics related to old age and aging are increasingly present.

Despite this growing interest in age related questions, which is currently developing in different subdisciplines, we have observed that research from other sub-disciplines or language specific approaches is often ignored and that invisible barriers seem to exist between the related scholarly cultures. Encouraging new approaches are reflected in the "call for the full integration of older adults into the variationist research agenda" (Pichler et al. 2018: 2) or in an increasing number of panels or papers held, e.g., at the bi-annual meetings of the International Pragmatics Association (IPrA) or other conference series in interactional linguistics, psycholinguistics or sociolinguistics.

Accordingly, it seems to be important to allow for collaborations across these barriers and to make agerelated topics in linguistics more visible not just as part of linguistic-related disciplines, but in their own right: The broad label "aging research" invites experts with different backgrounds, working on different languages, and provides a platform to connect them. The special collection, for which we want to thank Linguistics Vanguard and especially Suzanne Evans Wagner for their support, provides us with insights from different disciplines.

In this short introduction, we want to highlight some important aspects of the frameworks, data and languages of the individual contributions. The research is in many cases, if not in all, based on corpora - originating from different settings of intra- or inter-generational communication. As participants and subjects of the various investigations, individuals aging healthily are included as well as people with dementia (PwD) or other age-related diseases. In sociolinguistic and interactional research, up until now, tests for cognitive and somatic health have been rarely used. Bringing psychologically and medically trained researchers and

*Corresponding author: Annette Gerstenberg, University of Potsdam, Romance Linguistics, Am Neuen Palais 10, 14469 Potsdam, Germany, E-mail: gerstenberg@uni-potsdam.de Camilla Lindholm: University of Helsinki, Department of Finno-Ugrian and Scandinavian Studies, P.0. Box 2400014 University of Helsinki, Helsinki, Finland, E-mail: camilla.lindholm@helsinki.fi 
linguists together can help stimulate cooperation in developing and applying new approaches and can help answer the question, what kind of tests and anamnestic tools, and in which context, are useful. At the same time, besides the potentially negative effects of tests on the performance of older adults (for the ongoing discussion on the validity of tests see White et al. 2018), it has to be asked why exclusively older speakers must prove cognitive and somatic health, while the normality of middle-aged adults is rarely challenged. Finally, genuine linguistic definition criteria of normal aging are under development: language development remains dynamic in later life, and reducing this dynamic to what is perceived as decline risks falling short of acknowledging its complexity (Gerstenberg 2015). After a brief overview on the frameworks represented in the sections, the nature of the data and the languages and cultures tackled in the contributions, we will come back to these perspectives.

The papers are organized in four sections. The articles of the first section fruitfully connect the dynamics of language change with individual speakers' lifespan trajectories, with a focus on the extent to which individuals participate in this change. Lynn Anthonissen and Peter Petré (Grammaticalization and the linguistic individual. New avenues in lifespan research) trace grammatical change as reflected in a corpus of Early Modern English. David Bowie (Individual variation in the development of the Western Vowel System of Utah) is concerned with phonological change in the $20^{\text {th }}$ century, as are Johanna Mechler and Isabelle Buchstaller ([In]stability in the use of a stable variable) who continue the investigation up until the first decades of the $21^{\text {th }}$ century.

The second section features contributions which compare linguistic features in healthy older adults vs. older adults with impairments, ranging from mild cognitive impairment (MCI) to dementia, or contributions which develop a comparison between the patterns found in an older vs. younger age group, as in Dagmar Bittner's contribution (Implicit Causality in German students and seniors). Bittner sheds new light on the question of whether world knowledge or linguistic features provide a better explanation for the implicit causality bias. Daniel Müller-Feldmeth, Katharina Ahnefeld and Adriana Hanulíková (Processing of gender stereotypes in dementia and healthy older adults: A self-paced reading study) find that gender stereotypes as part of world knowledge are activated in PwD as well as in a healthy older control group. Confronted with the challenge of evaluating a large and highly valued corpus of longitudinal data, Claudia Frankenberg and Johannes Schröder (Perplexity - a new predictor of cognitive changes in spoken language? - results of the Interdisciplinary Longitudinal Study on Adult Development and Aging (ILSE)) present a measure used in automatic speech processing which could be applied to early detection of dementia.

The third section deals with theories originating from discourse analysis. The included papers apply these frameworks to seemingly mundane communication involving older members of the speech community. Anna Charalambidou (Repairs and old-age categorisations: Interactional and categorisation analysis) reconstructs the use of age categories in the interaction of older Cypriot women, Rachel Heinrichsmeier (Ageism and interactional (mis)alignment: Using microdiscourse analysis in the interpretation of everyday talk in a hair-salon) observes phenomena of everyday ageism in intergenerational communication. The starting point of Yoshiko Matsumoto's article (Taking the stance of quotidian in talking about pains: Resilience and defiance) is the potentially face threatening effect for older people who reveal physical weaknesses.

The fourth section explores how discourse analytical frameworks can provide an interesting perspective on data from health care communication contexts (cf. Lindholm 2016). Peter Backhaus' article No time to care? Interactional hurriedness in Japanese eldercare highlights a conflict between the initial statement "care takes time" and the manner in which practical care work is performed. Based on audio recordings of morning activities in a Japanese care facility, the researcher observes how hurriedness impedes a smooth and well-functioning care system. Heidi Hamilton and Adrienne R. Isaac (Agency and epistemic authority in question-answer sequences between museum guides and visitors diagnosed with Dementia in an art museum) analyze twelve video-recorded guided tours at a major art museum in Australia with visitors who have been diagnosed with dementia. Their study reveals how the museum visitors display agency and epistemic authority by asking questions and answering questions in their knowledge domain. Jan Svennevig and Anne Marie Landmark's article Accounting for forgetfulness in dementia interaction examines how PwD and their co-participants account for the PwD's lack of knowledge through the use of practices of normalizing, 
exceptionalizing and justifying the forgetfulness. Finally, Kaarina Mononen (Embodied care: touch in constructing affiliation and friendly relationship in an old people's home) examines how professional caregivers use touch in interaction with residents in a care facility. Using the methodological framework of interactional sociolinguistics, Mononen demonstrates how effectively touch is used to construct socio-emotional relationships.

In what follows, we comment on four cross-sectional topics, that is, on the nature of research data, theoretical frameworks, languages, and situational settings investigated in the presented papers. Thanks to an ever-increasing availability of data and a more creative approach towards compiling samples for panel studies, language change can be investigated with regard to individual trajectories and language development through adulthood. Taken seriously, this approach affects a basic assumption of diachronic linguistics: as stated by Anthonissen and Petré, only if the stability of the individual speakers' usage patterns can be demonstrated, can valid statements on diachronic change be made. The size of samples used in panel studies allows for a deeper understanding of individual circumstances, personalities and conditions for the adoption or rejection of linguistic innovations. Based on these case studies, existing hypotheses can be challenged and modified. Anthonissen and Petre examine three innovations that are key to the first grammaticalization of be going to in $17^{\text {th }}$ century English and find examples for the adoption of the most innovative variants even in older speakers of the first generation in the sample, who had reached adulthood before these innovations started spreading. Bowie investigates three phenomena of vowel change in the Western Vowel System of Utah over a period of 20-40 years. In the discussion of the results, he suggests that instability in individual trajectories may be indicative for an early stage of change not yet spread across the wider community. The longitudinal findings of Mechler and Buchstaller, which span more than 40 years, refine sociolinguistic information on the conditions for the participation in language change. They tackle the notion of age-grading on the example of the use of a variable that is perceptually linked to lower education, casual speech and younger speakers on the example of the realization of -ing.

Panel studies are also conducted in order to identify early indicators for MCI or dementia, as in the case of Frankenberg and Schröder who compare two stages of the ILSE samples, ten years apart. The data was processed with Automatic Speech Recognition (ASR). The indicator "perplexity" measures how accurately the model can make predictions on an unseen utterance produced by the participants in the study. The authors consider perplexity to be a promising indicator linked to decreased linguistic complexity and therefore a plausible early sign of cognitive impairment. Bittner demonstrates how the comparison of language production in an older vs. younger age group can help to better understand the usage patterns of implicit causality structures. The results shed new light on the question regarding which linguistic facets are crucial for the choice of the individual variants and on the interplay between world knowledge and linguistic structure. The linguistic interest of Müller-Feldmeth, Ahnefeld and Hanulíková concerns the relation between semantics and world knowledge as reflected in the activation of gender stereotypes. The comparison of PwD with a healthy control group provides further evidence that social knowledge is presented differently than other components of semantics.

Regarding the theoretical frameworks, the common denominator shared by the contributions from Charalambidou, Heinrichsmeier and Matsumoto is that their studies demonstrate how phenomena manifest themselves in naturally occurring interaction. The system of age category terms reconstructed by Charalambidou based on repair mechanisms in the interaction of older Cypriot women is particularly fine-grained. She demonstrates how a combination of membership categorization and conversation analysis is needed to investigate how age categorizations are shaped in interaction (Garfinkel 1967). Heinrichsmeier also investigates the mechanisms of everyday interaction at a micro-level. Combining conversation analysis and ethnography, she studies small talk in a hair salon to explore the subtle and often unnoticed practices of everyday ageism (Bytheway et al. 2007). Matsumoto focuses on how older storytellers use quotidian reframing in narratives about painful events. It is demonstrated how speakers use reframing to re-position themselves as resilient in life and to oppose negative sociocultural narratives associating old age with frailty and gloom.

Both Isaac and Hamilton's and Svennevig and Landmark's studies involving participants with dementia utilize the work by Heritage (2012) on social epistemics. Isaac and Hamilton's investigation of questionanswer sequences in museum settings generates results with clinical implications: the authors suggest that 
the physical environment could be systematically used in care facilities to spark discussions, promote individual agency and thereby increase the well-being of persons with dementia. The piece by Svennevig and Landmark focuses on potentially problematic and face-threatening moments in interaction involving PwD, that is, when PwD demonstrate a lack of knowledge in situations when their personal experiences are made relevant. The authors use the notion of account, more specifically: excuses and justifications, following Scott and Lyman (1968) and recent approaches such as Robinson (2016), for their analysis of different strategies to deal with forgetfulness. Their study provides a detailed report of a hitherto under-researched area of great practical relevance.

Drawing on the framework of interactional sociolinguistics (Gumperz 2001), Mononen investigates touching as a special resource in the sense of Goodwin (2000). Previous research on touch in interaction has mainly focused on situations involving adults and caregivers, and Mononen's account of touch as a means to construct affiliation in the care of older people contributes to a relatively new field of research on embodied interaction in the domain of health care. Backhaus dedicates his study to hurriedness as an under-researched topic in the field of communication in the care of older people (see Grainger 2004). By investigating turnrepetition and overlap as indicators, Backhaus studies the phenomenon of "hurriedness" at a micro-level. In conclusion, the author presents the result that working in a rushed manner does not necessarily speed things up, which is a finding with clear clinical implications.

Concerning the need for linguistic diversity in language and aging research, the included languages and the related cultures highlight the limits of the special collection, as it is restricted to Global North contexts, though a certain range of languages and varieties is presented. Data come from six different languages, in alphabetical order, Cypriot Greek (Charalambidou), English (Anthonissen and Petré; Heinrichsmeier - British English; Bowie; Mechler and Buchstaller - American English; Hamilton and Isaac - Australian English; Svennevig and Landmark - English of bilinguals), Finnish (Mononen, also with regard to body language), German (Bittner, Frankenberg and Schröder: Müller-Feldmeth, Ahnefeld and Hanulíková), Japanese (Backhaus, Matsumoto), and Norwegian (Svennevig and Landmark). Especially in this regard of represented languages and varieties, we consider the research program to be a starting point rather than a milestone. On the network's website (CLARe), the presentation of age-related corpora includes some more languages such as sign language, Belgian and hexagonal French, and Latin-American Spanish. The website represents an ongoing project and the languages featured will be enriched as a result of future conferences and network activities.

The linguistic varieties included in the papers of the special issue cover different facets of the standard varieties marked by different linguistic stages in the process of language change (Anthonissen and Petré, Bowie - within the Western Vowel System of Utah) or sociolinguistically meaningful variants (Mechler and Buchstaller). The question of the cultural conditions being examined is sometimes implicitly (Matsumoto) and sometimes explicitly (Charalambidou) addressed. The confrontation of these nuanced examples of historically, geographically and socially or culturally shaped data sets may contribute to raising the awareness of the crucial question to what extent linguistic data can tell us something on aging vs. how much they are simply the result of specific linguistic environments.

The network's corpus approach is meant to contribute to this awareness, as much attention is paid to the origin of the data sets. Four studies in the special issue are concerned with aging processes over time. Only one of the data sets used in these papers was originally designed for such an approach: the ILSE study was designed as longitudinal analysis. Frankenberg and Schroeder include a sample with two data points ten years apart. Three contributions present convincing strategies to develop a panel study approach by adjusting existing data sets. Mechler and Buchstaller analyze a panel study started in 1971 with six speakers (21-32 years old), and pursued in 2013, with the same speakers. The results are compared with findings from the United States. Bowie explores a collection of semi-extemporaneous religious sermons publicly broadcast over radio and television from Salt Lake City, from 1930 to present day; individuals appear multiple times over the course of decades, certainly not aware of the fact that, some decades apart, their speech would be linguistically analyzed. Anthonissen and Petré have built the corpus Early Modern Multiloquent Authors (EMMA), which includes 50 prolific writers born in the $17^{\text {th }}$ century, with their writings taken from EEBO and ECCO databases. 
The German data investigated in Frankenberg and Schröder have been gathered in interview settings, where the participants are taking the medical and psychological test series of the Heidelberg Longitudinal study (ILSE). The research presented by Müller-Feldmeth, Ahnefeld and Hanulíková was conducted in a nursing home, using different methods such as reaction times in a self-paced reading setting, combined with $\mathrm{Y} / \mathrm{N}$-questions on comprehension and a digit-span task. Bittner used sentence completion tasks which were done in an environment familiar to the participants; students were contacted at their university, and older people in institutions they frequented.

The situational settings for audio and video recordings form a part of the participants' life. They are unsupervised in the case of Charalambidou. In the hair salon where Henrichsmeier recorded the data of her analysis, an encounter of different generations takes place on a regular basis. This means that the case study she presents is significant for this specific environment. Video data are used by Hamilton and Isaac (in a museum) as well as by Mononen (in a nursing home), completed with field notes from the researchers' observations, and background interviews with the charge nurse. A similar mix of data collection methods is used for embedding the video-taped interactions of Svennevig and Landmark who recorded naturally occurring interactions as well as interviews and conversations with the researchers. The audio recordings of Backhaus capture morning activities in a Japanese care facility, in the northern outskirts of larger Tokyo metropolitan area.

In the cases where permission has been granted, the authors illustrate their contributions with audio samples. This approach is just a first step in the direction of a complete documentation of all analyzed data together with the resulting research papers. The case of Carolina Conversations Collection (Pope and Davis 2011) is an example of how the needs of complete medical data can be met and how ethical issues can be solved.

The wide range of factors with an impact on language use of older adults will continue to stimulate research on language and aging, especially when approaches from different backgrounds such as those represented in the special issue are taken into account. The CLARe network's activities have already resulted in new forms of cooperation. This starting point can be considered as an increase in awareness in all respects: awareness of the possible effects of language change when comparing older and younger adults in psycholinguistic research, awareness of the limits of what can be said about linguistic aging when considering the specificity of varieties, generations and cultures, awareness of possible impacts of cognitive and somatic aging when analyzing sociolinguistic variation, and awareness of what happens on the level of interaction when older speakers participate in tests and elicitation experiments which per se can be experienced as face threatening acts.

With the special issue, we present the somewhat paradoxical wish that a special collection could contribute to making the featured topics more commonplace, and to therefore reinforce the point that older people are an integral part of the speech community. The different contributions in the special collection show how established frameworks can be challenged and enriched when applied to age related data. Research on these topics invites us to re-think and to innovatively adapt linguistic frameworks and methods. Language and aging research highly deserve an increase in dynamics and visibility.

Acknowledgement: The authors wish to thank Freya Hewett for her assistance in the editing process.

\section{References}

Bytheway, Bill, Richard Ward, Caroline Holland \& Sheila Peace. 2007. Too old. Older people's accounts of discrimination, exclusion and rejection. A report from the research on age discrimination project (road) to help the aged. London: Open University and Help the Aged.

CLARe = Corpora for Language and Aging Research. 2014-2019. http://www.clare-corpora.org. Garfinkel, Harold (ed.). 1967. Studies in ethnomethodology. Englewood Cliffs, N.J.: Prentice-Hall.

Gerstenberg, Annette. 2015. A sociolinguistic perspective on vocabulary richness in a seven-year comparison of older adults. In Annette Gerstenberg \& Anja Voeste (eds.), Language development: The lifespan perspective (Impact: Studies in language and society Volume 37), 109-127. Amsterdam: Benjamins. 
Goodwin, Charles. 2000. Action and embodiment within situated human interaction. Journal of Pragmatics 32. 1489-1522. Grainger, Karen. 2004. Communication and the institutionalized elderly. In J.F. Nussbaum \& J. Coupland (eds.), Handbook of communication and aging research (2nd edn.), 479-497. Mahwah \& London: Erlbaum.

Gumperz, John J. 2001. Interactional sociolinguistics. A personal perspective. In Deborah Schiffrin, Deborah Tannen \& Heidi Hamilton (eds.), The handbook of discourse analysis, 215-228. Oxford: Blackwell Publishers.

Heritage, John. 2012. Epistemics in action: Action formation and territories of knowledge. Research on Language and Social Interaction 45. 1-29.

Lindholm, Camilla. 2016. Boundaries of participation in care home settings: Use of the Swedish token jaså by a person with dementia. Clinical Linguistics and Phonetics 30(10). 832-848.

Pichler, Heike, Suzanne E. Wagner \& Ashley Hesson. 2018. Old-age language variation and change: Confronting variationist ageism. Language and Linguistics Compass 12(6). 1-21.

Pope, Charlene \& Boyd H. Davis. 2011. Finding a balance: The Carolinas conversation collection. Corpus Linguistics and Linguistic Theory 7(1). 143-161.

Robinson, Jeffrey D. 2016. Accountability in social interaction. Oxford: Oxford University Press.

Scott, Marvin B. \& Stanford M. Lyman. 1968. Accounts. American Sociological Review 33. 46-62.

White, Naomi, Larnee Flannery, Alice McClintock \& Liana Machado. 2018. Repeated computerized cognitive testing: Performance shifts and test-retest reliability in healthy older adults. Journal of Clinical and Experimental Neuropsychology 41(2). 179-191. 